\title{
SARS-CoV-2 Rapid Antigen Detection in Respiratory and Nonrespiratory Specimens in COVID-19 Patients
}

\author{
Vandana Vijayeta Kiro ${ }^{1}$ Parul Singh ${ }^{1}$ Sharad Srivastav ${ }^{20}$ Richa Aggarwal ${ }^{3} \quad$ Kapil Dev Soni $^{3}$ \\ Yudhyavir Singh ${ }^{3}$ Abhishek Singh ${ }^{3}$ Anjan Trikha ${ }^{3}$ Purva Mathur ${ }^{4}$
}

${ }^{1}$ Department of Microbiology, Jai Prakash Narayan Apex Trauma Center (JPNATC), All India Institute of Medical Sciences, New Delhi, India

2 Junior Research Fellow Program, Jai Prakash Narayan Apex Trauma Center (JPNATC), All India Institute of Medical Sciences, New Delhi, India

${ }^{3}$ Department of Anaesthesia and Critical Care, All India Institute of

Medical Sciences IIMS, New Delhi, India

${ }^{4}$ Division of Clinical Microbiology, Department of Laboratory

Medicine, Jai Prakash Narayan Apex Trauma Center (JPNATC),

All India Institute of Medical Sciences, New Delhi, India

J Lab Physicians 2022;14:369-372.
Address for correspondence Purva Mathur, MD, Division of Clinical Microbiology, Department of Laboratory Medicine, JPNATC, AlIMS, New Delhi, 110029, India (e-mail: purvamathur@yahoo.co.in).

\author{
Abstract \\ Keywords \\ - COVID-19 \\ - SARS-CoV-2 \\ - rapid antigen test \\ - nasopharyngeal \\ - nonrespiratory \\ samples
}

Rapid antigen testing for coronavirus disease 2019 (COVID-19) available at present provides immediate results at low cost with less expertise and without any need of sophisticated infrastructure. Most of these test kits available are for nasopharyngeal samples. This is a novel study to detect the presence of COVID antigen in samples other than throat and oropharyngeal. Various samples received from patients admitted in the COVID-19 dedicated center were tested for the presence of antigen. Same procedure was followed as done for the nasopharyngeal sample. A total of 150 samples were tested, which included ascitic fluid, pleural fluid, drain fluid, bile, bronchoalveolar lavage, cerebrospinal fluid, endotracheal tube aspirate, sputum, tissue, and urine. Out of $150,11(7.33 \%)$ were positive and 138 (92.66\%) were negative for the antigen test. The COVID-19 antigen test kit, though designed for nasopharyngeal samples, was able to detect the presence of antigen in other clinical samples.

\section{Introduction}

A novel human pathogen emerged at the end of 2019 at the city of Wuhan, China and is now known as severe acute respiratory syndrome coronavirus 2 (SARS-CoV-2), causing the coronavirus disease 2019 (COVID-19). ${ }^{1}$ In COVID-19, fever is usually the first symptom with subsequent development of respiratory symptoms. ${ }^{2,3}$ Most patients with COVID19 experience a mild disease course; albeit, approximately $20 \%$ develop severe disease with high mortality rate. ${ }^{2,3}$

published online February 9, 2022
DOI https://doi.org/

10.1055/s-0042-1742415. ISSN 0974-2727.
Laboratory diagnosis and management of COVID-19 has been helpful in combating the spread of SARS-CoV-2. At present, the gold standard for COVID-19 diagnosis is reverse transcription-quantitative polymerase chain reaction (RTqPCR) which uses nasopharyngeal swabs, throat swabs, or saliva samples. ${ }^{4}$ RT-qPCR kits that do not require viral ribonucleic acid (RNA) extraction and high-throughput RTqPCR systems have also been developed. Although such tests are widely utilized in tertiary care centers and large wellequipped hospitals, they are rarely available in the local (c) 2022. The Indian Association of Laboratory Physicians. All rights reserved.

This is an open access article published by Thieme under the terms of the Creative Commons Attribution-NonDerivative-NonCommercial-License, permitting copying and reproduction so long as the original work is given appropriate credit. Contents may not be used for commercial purposes, or adapted, remixed, transformed or built upon. (https://creativecommons.org/ licenses/by-nc-nd/4.0/)

Thieme Medical and Scientific Publishers Pvt. Ltd., A-12, 2nd Floor, Sector 2, Noida-201301 UP, India 
clinics which are more approachable for the patients who are under suspicion.

Studies done on rapid antigen tests (RATs) have shown sensitivity of $61.70 \%$ and specificity of $98.26 \%$ for diagnosis of COVID-19. ${ }^{5}$ In the present study, we have used RAT to detect SARS-CoV-2 antigen in samples other than nasopharyngeal.

\section{Material and Methods}

This descriptive study was done at a single center dedicated for COVID-19 patients in a tertiary care center in North India. RAT was done on samples like ascitic fluid, pleural fluid, drain fluid, bile, bronchoalveolar lavage (BAL), cerebrospinal fluid, endotracheal tube aspirate (ETA), sputum, tissue, and urine, which were sent to the microbiology laboratory for culture and sensitivity testing. The RAT was done according to the kit insert provided by the STANDARD Q COVID-19 antigen test which is ideally for testing nasopharyngeal sample. Samples were used directly without any dilution. Test was performed as soon as the sample was received and result noted within 30 minutes of the test.

\section{Ethical Approval}

Since the samples consisted of routine samples for culture, ethical consent was not taken. However, a blanket ethical clearance for evaluation of all COVID antigen kits was obtained having the ethical committee approval number IEC 668/ July 3, 2020.

\section{Result}

A total of 150 patients were included in the study admitted in the hospital. Out of 150 patients, 88 (58.66\%) were male and 62 (41.33\%) were female. Patients before admission were confirmed as having COVID-19 disease by RT-PCR (73), Truenat Beta (14), Xpert (20), antigen test (17), Xpert and antigen (1), RT-PCR and antigen (6), Truenat and RT-PCR (17), and Xpert and RT-PCR (2). Samples other than nasopharyngeal swab collection were tested. - Table 1 shows the distribution of sample and their test results. Eleven (7.33\%) samples were positive and 138 (92.66\%) were negative for the test. Out of the 11 specimens which were positive for the antigen test, 7 were respiratory, 3 were fluid, and 1 was bile. The duration of illness was 6.2 days ( $95 \% \mathrm{CI}$ : 3.6-8.7) for the majority of patients with positive specimens. Exceptions were there as for one sample (BAL) test was positive after 2 weeks of onset of symptoms and for another (sputum) patient was asymptomatic. - Fig. 1 shows the time interval for each positive sample depicting starting of symptoms to the test.

\section{Discussion}

Since the outbreak of COVID-19, various strategies are being tried, tested, and followed for the rapid detection, treatment, and containment of this disease. Many have lost their life due to this pandemic. Globally, 101,636,470 have been infected and 2,194,790 have died. ${ }^{6}$
Table 1 Clinical samples with severe acute respiratory syndrome coronavirus 2 (SARS-CoV-2) antigen test results

\begin{tabular}{|l|l|l|}
\hline Samples & $\begin{array}{l}\text { Antigen test } \\
\text { positive/negative }\end{array}$ & Total \\
\hline Ascitic fluid & $2 / 11$ & 13 \\
\hline Drain fluid & $1 / 6$ & 7 \\
\hline Pleural fluid & $0 / 4$ & 4 \\
\hline Cerebrospinal fluid & $0 / 6$ & 6 \\
\hline Bile & $1 / 3$ & 4 \\
\hline Urine & $0 / 74$ & 74 \\
\hline Tissue & $0 / 1$ & 1 \\
\hline Sputum & $1 / 6$ & 7 \\
\hline Endotracheal aspirate & $4 / 26$ & 30 \\
\hline Bronchoalveolar lavage & $2 / 2$ & 4 \\
\hline Total & $11 / 139$ & 150 \\
\hline
\end{tabular}

In our country, till now 10,727,240 have suffered and 154,069 have succumbed to this disease. ${ }^{6}$ As far as diagnostics is considered, at present rRT-PCR is considered the gold standard. To minimize infrastructure cost, various other modes of nucleic acid detection modalities like Truenat and CBNAAT are also in use. Antigen testing with high specificity ${ }^{5}$ has also helped in rapid detection, isolation, and treatment of individuals suspected of having COVID-19. RATs for SARS-CoV2 are being usually done in throat and nasopharyngeal sample. In a study done in Japan, samples other than nasopharyngeal and throat were also tested with RT-PCR and four different kits of rapid antigen for SARS-CoV-2 were evaluated. ${ }^{6}$ Sample tested were gargle lavage, saliva, throat swab, nasal vestibule swab, nasopharyngeal, sputum, and tracheal aspirate. Swabs were transported in BD universal viral transport medium; saliva, sputum, and tracheal aspirate samples were diluted in $\mathrm{BD}$ universal viral transport medium if needed and were used as test specimens. Gargle lavages were tested directly. Among the four antigen test kits, one kit was same as what we had used. That kit was able to identify SARS-CoV-2 antigen in saliva (10/27), nasopharyngeal swab (8/18), sputum (1/4), and tracheal aspirate (7/17). In our study, the kit detected the antigen in endotracheal aspirate (4/29), BAL (2/4), sputum (1/7), ascitic fluid (2/13), drain fluid (1/7), and bile (1/4).

With regard to detection of SARS-CoV-2 in nonrespiratory samples, studies have shown its presence in various nonrespiratory samples. RT-PCR has detected SARS-CoV-2 RNA in stool sample in many studies. ${ }^{7-12}$ In some studies, where serum or blood were tested, the viral RNA was detected in 30 to $87.5 \%$ of patients with COVID-19. The viral RNA shedding was for longer duration in intensive care unit patients $(14.63 \pm 5.88$ days standard deviation [SD]) compared with non-ICU patients $\left(10.17 \pm 6.13\right.$ days SD). ${ }^{13-16}$ This viral RNA has also been detected in ocular tissue too. ${ }^{17}$ Ours is a novel study on antigen detection on samples other than nasopharyngeal with positivity of $7.33 \%$. Our study shows that viral antigen and therefore the virus may be present in many other organs apart from the respiratory tract. 


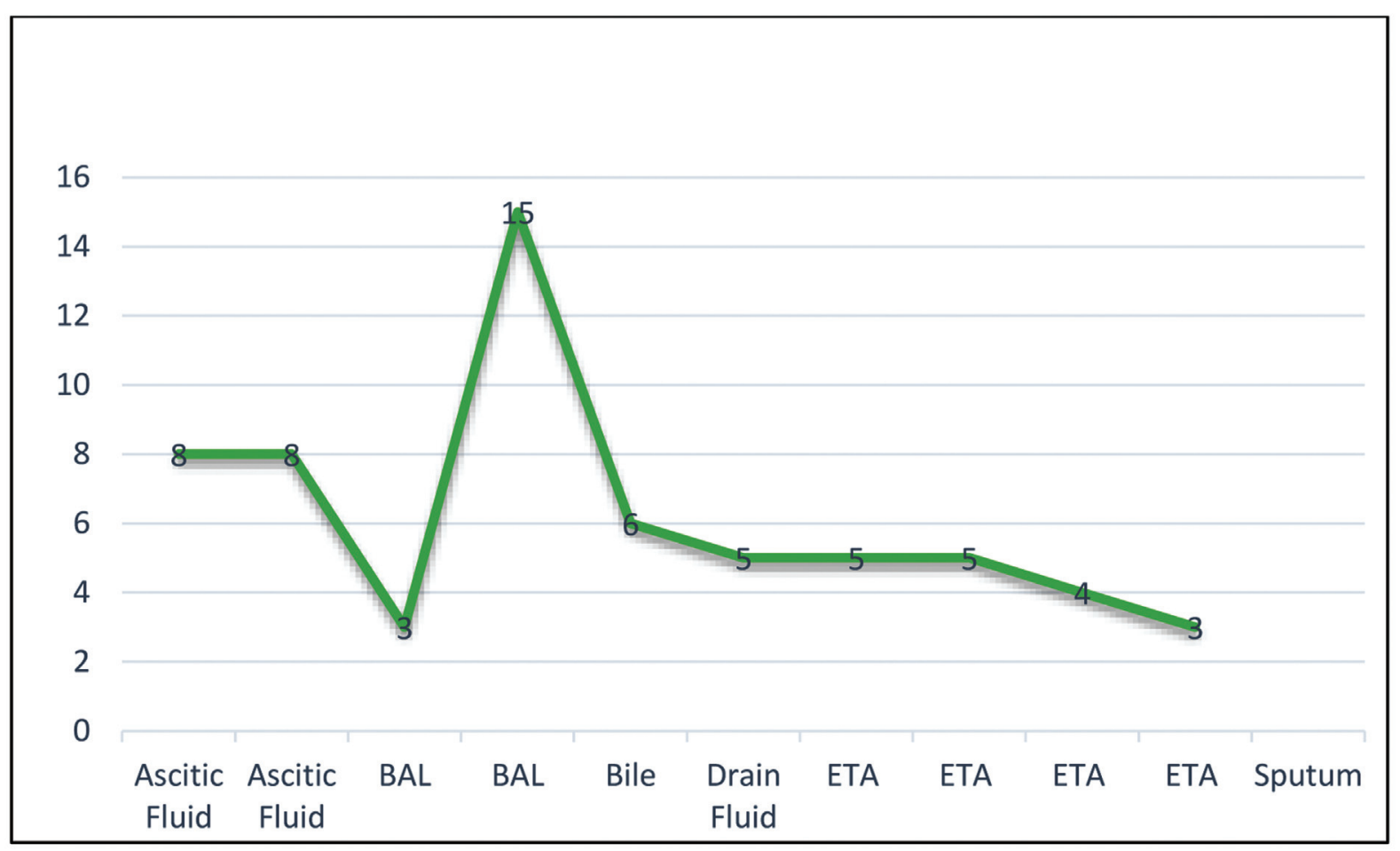

Fig. 1 Time interval from symptoms to the test (days).

\section{Conclusion}

The COVID-19 antigen test kit, though dedicated for nasopharyngeal samples was able to detect presence of antigen in other clinical samples. The sample infectivity and transmissibility albeit cannot be assessed with this test alone.

\section{Funding \\ No external funding was received. \\ Conflict of Interest \\ All the authors report no conflict.}

\section{Acknowledgment}

The authors would like to thank all participating employees from the JPNATC, AIIMS, Delhi for their help with sample collection, processing, and data collection.

\section{References}

1 Zhu N, Zhang D, Wang W, et al; China Novel Coronavirus Investigating and Research Team. A novel coronavirus from patients with pneumonia in China, 2019. N Engl J Med 2020;382(08): 727-733

2 Huang C, Wang Y, Li X, et al. Clinical features of patients infected with 2019 novel coronavirus in Wuhan, China. Lancet 2020;395 (10223):497-506

3 Wang D, Hu B, Hu C, et al. Clinical characteristics of 138 hospitalized patients with 2019 novel coronavirus-infected pneumonia in Wuhan, China. JAMA 2020;323(11):1061-1069

4 Sethuraman N, Jeremiah SS, Ryo A. Interpreting diagnostic tests for SARS-CoV-2. JAMA 2020;323(22):2249-2251
5 Yamayoshi S, Sakai-Tagawa Y, Koga M, et al. Comparison of rapid antigen tests for COVID-19. Viruses 2020;12(12):1420

6 Accessed December 13, 2021: https://covid19.who.int. Last updated; January 29, 2021

7 Xiao F, Sun J, Xu Y, et al. Infectious SARS-CoV-2 in feces of patient with severe COVID-19. Emerg Infect Dis 2020;26(08):1920-1922

8 Zhang N, Gong Y, Meng F, et al. Comparative study on virus shedding patterns in nasopharyngeal and fecal specimens of COVID-19 patients. Sci China Life Sci 2020;64(03):486-488

9 Chen Y, Chen L, Deng Q, et al. The presence of SARS-CoV-2 RNA in the feces of COVID-19 patients. J Med Virol 2020;92(07):833-840

$10 \mathrm{Wu}$ Y, Guo C, Tang L, et al. Prolonged presence of SARS-CoV-2 viral RNA in faecal samples. Lancet Gastroenterol Hepatol 2020;5(05): 434-435

11 Zhao F, Yang Y, Wang Z, Li L, Liu L, Liu Y. The time sequences of respiratory and rectal viral shedding in patients with coronavirus disease 2019. Gastroenterology 2020;159(03):1158-1160.e2

12 Zheng S, Fan J, Yu F, et al. Viral load dynamics and disease severity in patients infected with SARS-CoV-2 in Zhejiang province, China, January-March 2020: retrospective cohort study. BMJ 2020;369: $\mathrm{m} 1443$

13 Ling Y, Xu SB, Lin YX, et al. Persistence and clearance of viral RNA in 2019 novel coronavirus disease rehabilitation patients. Chin Med J (Engl) 2020;133(09):1039-1043

14 Fang Z, Zhang Y, Hang C, Ai J, Li S, Zhang W. Comparisons of viral shedding time of SARS-CoV-2 of different samples in ICU and nonICU patients. J Infect 2020;81(01):147-178

15 Zhang W, Du RH, Li B, et al. Molecular and serological investigation of 2019-nCoV infected patients: implication of multiple shedding routes. Emerg Microbes Infect 2020;9(01):386-389

16 Hogan CA, Stevens BA, Sahoo MK, et al. High frequency of SARSCoV-2 RNAemia and association with severe disease. Clin Infect Dis 2021;72(09):e291-e295

17 Jones KM, Titus MS, Dennis E, Hicks E, Majmudar PA, Kumar A, Mian SI. Prevalence of SARS-CoV-2 in human post-mortem ocular tissues. Ocul Surf 2021;19:322-329. Doi: 10.1016/j.jtos.2020.11.002 